\title{
Sensores de fibra óptica FBG para el monitoreo de la salud estructural de los puentes
}

\author{
Structural Health Monitoring of Bridges \\ with Fiber Bragg Grating Sensors
}

Francisco Navarro-Henríquez'

Fecha de recepción: 19 de febrero del 2014

Fecha de aprobación: 27 de abril del 2014

Navarro-Henríquez, F. Sensores de fibra óptica FBG para el monitoreo de la salud estructural de los puentes. Tecnología en Marcha. Vol. 27,

N 4, Octubre-Diciembre. Pág 3-13. 


\section{Palabras clave}

Rejilla de fibra Bragg; sensores de fibra óptica FBG; monitoreo de salud estructural; interrogador óptico; sensores FBG de esfuerzo y vibración.

\section{Resumen}

El presente artículo forma parte de un estudio inicial realizado como parte del proyecto de investigación "eBridge 2.0: sistema integrado para el desempeño de puentes".

Los sistemas con sensores de fibra óptica FBG (Fiber Bragg Grating) se han venido consolidando en el Monitoreo de la Salud Estructural de puentes, en Pruebas No Destructivas estáticas y dinámicas con mediciones de deformación, desplazamiento, deflexión, temperatura y vibraciones. En este trabajo se presenta una breve introducción a la tecnología y los fundamentos de los sensores de fibra óptica, su uso y las ventajas comparativas respecto a su contraparte tradicional. Como muestra práctica de la aplicación de sensores FBG, se describen sus características y se presentan gráficas de medición. Por último, se mencionan algunos aspectos clave que se deben tener en cuenta para su adecuado uso en el campo.

\section{Key words}

Fiber Bragg Grating; FBG fiber optics sensors; Structural Health Monitoring; Optical Interrogator; Strain and vibration FBG sensors.

\section{Abstract}

This paper describes part of the initial studies in the research project eBridge 2.0: integrated bridge system performance.

Systems with fiber optic sensors FBG (Fiber Bragg Grating) are consolidated in the Structural Health Monitoring (SMH) of bridges, Nondestructive Testing (NDT) static and dynamic measurements of deformation, displacement, deflection, temperature and vibration. This article provides a brief introduction to the technology and the fundamentals of fiber optic sensors, also present comparative advantages over its traditional counterpart is presented. Their characteristics are described and measurement graphics are presented as an application example of the FBG sensors. Finally, some key aspects to consider for proper use in the field are mentioned.

\section{Introducción}

La fibra óptica se ha utilizado históricamente en sistemas de comunicaciones que usan ondas luminosas para la transmisión y recepción de información. Pero desde inicios de la década de 1990 la investigación se ha extendido al campo de la optoelectrónica, lo que ha conllevado el desarrollo de productos en la industria de la fibra óptica, especialmente la tecnología de sensores (Kumar y Madhav, 20 I0).

La tecnología de sensores de fibra óptica utiliza este material como elemento sensor o como medio de transmisión de señales desde un sensor remoto hacia un dispositivo electrónico que las procesa. Junto con el desarrollo de productos confiables y de alta calidad, se ha dado una rápida aceptación, y en algunos casos incluso el reemplazo, de los tradicionales sensores eléctricos en una variedad de aplicaciones de detección tales como: mediciones de deformación, esfuerzo (strain), temperatura, vibraciones, desplazamiento y deflexión.

De manera específica, en el área de aplicación del Monitoreo de la Salud Estructural (SHM, por sus siglas en inglés) de obra civil, los sensores de fibra óptica han demostrado un gran potencial, ya que poseen una serie de ventajas únicas cuando se comparan con su contraparte tradicional.

La detección óptica usa luz en vez de electricidad y fibra óptica estándar en lugar de cable de cobre. Las fibras ópticas no son conductivas, son inmunes al ruido inducido por la interferencia electromagnética (EMI, por sus siglas en inglés) y los altos voltajes y resistentes a la corrosión metálica. 
También son capaces de transmitir datos a larga distancia, con escasa pérdida en la integridad de la señal. Asimismo, es posible interconectar múltiples sensores de fibra óptica con una sola fibra óptica para reducir significativamente el tamaño, el peso, la complejidad de los sistemas de medición y, potencialmente, a largo plazo el costo de mantenimiento.

A continuación se enumeran algunas características de los sensores de fibra óptica (National Instruments, 20 I I):

- No son conductivos.

- Son pasivos eléctricamente.

- Son inmunes a la EMI y al ruido inducido.

- Realizan mediciones a distancias muy grandes (hasta $10 \mathrm{~km}$ ).

- Se pueden interconectar múltiples sensores en una sola fibra óptica, sin ningún orden en particular y de diferentes tipos: esfuerzo, temperatura, acelerómetro, presión.

- No requieren calibración durante su vida útil, luego de la instalación inicial.

- Manejo simplificado del cable, ventajoso por lo liviano de la fibra óptica.

- Mayor durabilidad potencial de los sensores y de la instalación.

- Tiempo de respuesta más rápido que los sensores eléctricos equivalentes.

Los sensores de fibra óptica son una alternativa muy apropiada para aplicaciones que se distribuyen en una amplia zona geográfica. Decenas de sensores que incluyen deformación, esfuerzo, aceleración y temperatura pueden conectarse en cadena a lo largo de una sola fibra óptica. Los sensores ópticos son confiables para la instalación a largo plazo y como resultado se reduce el mantenimiento y la instalación del cableado requerido.

No obstante, en muchas de las aplicaciones del mundo real se requiere un enfoque híbrido, utilizando los beneficios de las tecnologías de medición tanto eléctricas como ópticas. La tecnología de los sensores de fibra óptica permite hacer mediciones que antes no se podían practicar, o eran incluso imposibles en algunos casos, con sensores convencionales.
Reseña de las tecnologías de los sensores de fibra óptica

En la actualidad, existe una variedad de tecnologías de sensores ópticos, entre las que se pueden mencionar los interferómetros Fabry-Perot, Fiber Bragg Gratings (FBG), sensores distribuidos sobre la base de Rayleigh, Raman y técnicas de dispersión óptica de Brillouin. Según la modulación de la luz y del efecto que se utiliza para medir los fenómenos físicos, estas tecnologías de detección óptica se pueden clasificar de la siguiente manera (National Instruments, 201 I):

- Intensidad

- atenuación de luz

- Interferómetro

- diferencia de fase entre dos ondas de luz (Sagnac, Michaelson, Mach-Zehnder)

- Resonante

- frecuencia de resonancia óptica de una cavidad óptica (Fabry-Perot)

- Distribuido

- retrodispersión (Rayleigh, Raman, Brillouin)

- Polarimétrico

- estado de polarización de la onda de luz

- Interferencia espectral

- frecuencia de onda de luz que interfiere con una estructura periódica (Fiber Bragg Grating)

Uno de los tipos de sensores ópticos más versátiles y ampliamente utilizados es la Rejilla de Fibra Bragg (FBG), la cual refleja una longitud de onda de luz específica que se transmite en respuesta a variaciones en temperatura y/o esfuerzo (strain).

Desde su descubrimiento por Ken Hill (1978) y su posterior desarrollo en varios centros de investigación, las rejillas dentro del núcleo de la fibra se han utilizado ampliamente en la industria de las telecomunicaciones. Sin embargo, dada su capacidad intrínseca para medir una gran cantidad de parámetros como el esfuerzo, la temperatura y la presión, junto con la flexibilidad de su uso, ya sea como sensor de un solo punto o como un arreglo de sensores multipunto, los elementos FBG han sido reconocidos desde sus inicios como ideales para el sensado de variables físicas. 
Con base en su topología y configuración, los sensores de fibra óptica pueden clasificarse como de Solo un punto, Multipunto o Distribuido. En la figural se comparan las distintas tecnologías mencionadas.

Los sensores FBG realizan la transformación directa del parámetro detectado, ya sea éste deformación, temperatura u otro, a longitudes de onda ópticas -independiente de los niveles de luz, conector o pérdidas de fibra- $u$ otros sensores FBG instalados a lo largo de la fibra óptica a diferentes longitudes de onda.

Esta tecnología puede hacer frente a una amplia gama de aplicaciones, debido a su capacidad para medir muchos tipos de fenómenos físicos y de la cadena en serie de múltiples sensores a lo largo de una sola fibra óptica. Esto hace que sea una tecnología ideal para la sustitución de sensores eléctricos convencionales.

\section{Fundamentos de los Sensores de Fibra Óptica FBG}

El espectro de frecuencias luminosas se puede dividir en tres bandas generales: (Tomasi, 2003):

- Infrarrojo

- Visible

- Ultravioleta

En la banda infrarroja las longitudes de onda de luz son demasiado grandes para que las vea el ojo humano. La banda visible contiene longitudes de onda de luz a las que responde el ojo humano. Las longitudes de onda de luz en la banda ultravioleta son demasiado cortas para que las vea el ojo humano.

El orden de las unidades de las frecuencias de las ondas luminosas se encuentra entre las decenas tera-hercios $\left(\mathrm{THz}-\mathrm{I} \times \mathrm{IO}^{12}\right)$ para luz infrarroja, hasta decenas de peta-hercios $\left(\mathrm{PHz}-\mid \times 10^{15}\right)$ para la luz ultravioleta, prefijos no comúnmente empleados para la unidad de frecuencia. Cuando se manejan ondas electromagnéticas de mayor frecuencia, como las luminosas, se acostumbra a usar unidades de longitud de onda y no de frecuencia.

La longitud de onda es la distancia que ocupa en el espacio un ciclo de una onda electromagnética. La longitud de onda depende de la frecuencia de la onda y de la velocidad de la luz. La longitud de onda tiene una relación inversa con la frecuencia, a mayor frecuencia, menor longitud de onda, y viceversa. La longitud de onda $\lambda$ (lambda) puede expresarse como:

$$
\lambda=\frac{c}{f}
$$

donde:
$\lambda$ : longitud de onda (metros/ciclo)
c: velocidad de la luz $(\approx 300,000,000$ metros por segundo)
$f$ : frecuencia (hercios)

\begin{tabular}{|l|l|}
\hline \multicolumn{1}{|c|}{ Topología } & \multicolumn{1}{|c|}{ Tecnologia (variables medidas) } \\
\hline Sensor solo un Punto (single point) & $\begin{array}{l}\text { - Fabry-Perot } \\
\text { (temperatura, presión). Rango }<10 \mathrm{~km}\end{array}$ \\
\hline Multi-Punto (Cuasi-Distribuido) & $\begin{array}{l}\text { - Fiber Bragg Grating }-\mathrm{FBG}- \\
\text { (esfuerzo, temperatura, aceleración, } \\
\text { presión, desplazamiento) Rango }<50 \mathrm{~km}\end{array}$ \\
\hline Sensado Distribuido & $\begin{array}{l}\text { Fibra Retrodispersión } \\
\text { - Rayleigh (esfuerzo, temperatura) }<70 \mathrm{~m} \\
\text { - Brillouin (esfuerzo, temperatura, } \mathrm{dB})<50 \mathrm{~km} \\
\text { - Raman (temperatura) rango }<20 \mathrm{~km}\end{array}$ \\
\hline
\end{tabular}

Figura I. Comparación de las tecnologías de sensores ópticos. 
Con las frecuencias luminosas, la longitud de onda se suele expresar en nanómetros ( $\mathrm{nm}$ equivale a I $\times 10^{-9}$ metros).

La figura 2 muestra como referencia el espectro luminoso indicando la longitud de onda en nanómetros $(\mathrm{nm})$ y la frecuencia correspondiente en tera-hercios $(\mathrm{THz})$.

Una fibra óptica está compuesta por tres elementos principales: el núcleo, el revestimiento y la capa protectora. El revestimiento refleja la onda de luz de regreso al núcleo, asegurando la transmisión de la luz en el núcleo. Esta acción es posible debido a un índice refractivo más alto en el núcleo con relación al revestimiento, lo que provoca una total reflexión interna de luz. La capa protectora sirve para proteger la fibra de condiciones externas y daños físicos (National Instruments, 20I0).

Un sensor de fibra óptica funciona al modular una o más propiedades de una onda de propagación de luz, incluyendo intensidad, fase, polarización y frecuencia, en respuesta a la variable física que se mide.

Los sensores de fibra óptica FBG se manufacturan aprovechando la tecnología actual existente en la industria de la fibra óptica para grado de telecomunicaciones, por medio de técnicas de exposición (Davis et al., 1996). Dos configuraciones típicas consisten en exponer una pequeña porción de la fibra óptica a dos rayos de luz ultravioleta (UV) que interfieren o un haz de luz UV enfocado a través de una máscara de fase. Esto crea en el núcleo de la fibra óptica una pequeña modulación periódica del índice de refracción. Esta alternación del índice refractivo de la fibra es permanente, dada la intensidad de luz a la que es expuesto. A la variación periódica que resulta en el índice refractivo se le llama fiber Bragg grating.

Debido a la naturaleza periódica de la perturbación creada del índice refractivo, solo algunas frecuencias ópticas discretas resonarán en la estructura.

$$
\lambda_{B}=2 \cdot n_{e f f} \cdot \Lambda
$$

Por lo tanto, cuando haces de luz de amplio espectro se envían por el núcleo del sensor de fibra óptica FBG, la energía incidente en la rejilla creada a una frecuencia resonante específica será reflejada de vuelta por la fibra óptica, dejando pasar sin ser afectado el resto del espectro óptico. La longitud de onda central de esta condición de resonancia en el sensor de fibra óptica FBG puede expresarse como: donde:

$\lambda_{B}$ : la longitud de onda Bragg

$n_{\text {eff }}$ : índice refractivo efectivo del núcleo de la fibra

$\Lambda$ : periodo de la modulación del índice refractivo o separación entre rejillas.

La figura 3 muestra en forma esquemática las características de transmisión y reflexión en los sensores de fibra óptica FBG.

Debido a que la longitud de onda Bragg $\left(\boldsymbol{\lambda}_{B}\right)$ es una función de la separación entre las rejillas, los sensores de fibra óptica FBG se pueden fabricar con varias longitudes de onda Bragg, lo que permite a diferentes sensores FBG reflejar longitudes de onda de luz específicas.

Nótese que cualquier cambio en la periodicidad de la modulación del índice refractivo o la distancia de separación entre las rejillas cambiará la longitud de onda Bragg $\left(\lambda_{B}\right)$. En consecuencia, cualquier temperatura o efecto de esfuerzo inducido (strain) en el sensor FBG podrán ser determinados por el correspondiente desplazamiento en la longitud de onda central Bragg reflejada.

El cambio en la longitud de onda $\lambda_{B}$ debido a un esfuerzo (strain) sobre el sensor de fibra óptica FBG y la temperatura está dado por:

$$
\frac{\Delta \lambda_{B}}{\lambda_{B}}=\left(1-p_{e}\right) \cdot \varepsilon+\left(\alpha_{\Lambda}+\alpha_{n}\right) \cdot \Delta T
$$

donde:

$\Delta \lambda_{B}$ : el cambio de la longitud de onda Bragg

$\lambda_{B}$ : la longitud de onda Bragg inicial

$p_{\mathrm{e}}$ : constante foto-eslástica efectiva para la fibra óptica

$\varepsilon=\Delta \mathrm{L} / \mathrm{L}$ : esfuerzo (strain) sobre la longitud del sensor FBG

$\alpha_{\Lambda}$ : coeficiente de expansión térmica. Cambio en índice refractivo por $\Delta T$.

$\alpha_{n}$ : coeficiente óptico térmico. Describe la expansión de la rejilla por $\Delta \mathrm{T}$.

$\Delta T$ : cambio de temperatura. 
El primer término de la expresión, $\left(I-p_{e}\right) \varepsilon$, describe el cambio que ocurre en la longitud de onda Bragg debido a la aplicación de un esfuerzo (strain) ya sea de tensión, compresión, flexión o torsión. El segundo término describe el impacto debido a la temperatura en el cambio de longitud de onda.

Un sistema de sensores de fibra óptica FBG se compone al menos de (Micron Optics, 20 I2):

- Fuente y detector de luz.

- Elementos sensores de fibra óptica FBG.

- Fibra óptica y accesorios para la interconexión de la fibra.

El principio del funcionamiento del sistema óptico consiste en que los elementos sensores de fibra óptica FBG modulan algún parámetro de la señal enviada desde la fuente de luz (intensidad, longitud de onda, polarización, fase), lo cual da lugar a un cambio en las características de la señal óptica recibida en el detector de luz.
La fuente y el detector de luz del sistema se incorporan en un equipo denominado Interrogador Óptico. Los métodos de interrogación óptica pueden categorizarse como: Multiplexación por División de Longitud de onda (WDM, siglas en inglés de Wavelength Division Multiplexing) y Multiplexación por División de Tiempo (TDM, siglas en inglés de Time Division Multiplexing).

Algunas alternativas tecnológicas para clasificar los interrogadores para sistemas de sensores de fibra óptica FBG son las siguientes:

- Fuente de Amplio Espectro, arreglo de Diodo (WDM)

- Espectrómetro Multiplexado por División de Tiempo (TDM)

- Reflectómetro óptico en Dominio de frecuencia (OFDR-WDM)

- Reflectómetro óptico en Dominio de tiempo (OTDR-TDM)

\begin{tabular}{|c|c|c|c|c|c|c|}
\hline \multicolumn{2}{|c|}{ Ultravioleta } & \multicolumn{3}{c|}{ Luz visible } & \multicolumn{2}{c|}{ Infrarojo } \\
\hline extremo & cercano & violeta & verde & rojo & cercano & lejano \\
\hline & & & & & & \\
\hline
\end{tabular}

\begin{tabular}{|l|c|c|c|c|c|c|c|c|}
\hline longitud de onda $(\mathrm{nm})$ & 10 & 300 & 390 & 492 & 622 & 770 & 6000 & 100000 \\
\hline frecuencia $(\mathrm{THz})$ & 30000 & 999 & 769 & 609 & 482 & 389 & 50 & 3 \\
\hline
\end{tabular}

Figura 2. Espectro luminoso $\lambda(\mathrm{nm})$ vrs frec.( $\mathrm{THz})$.

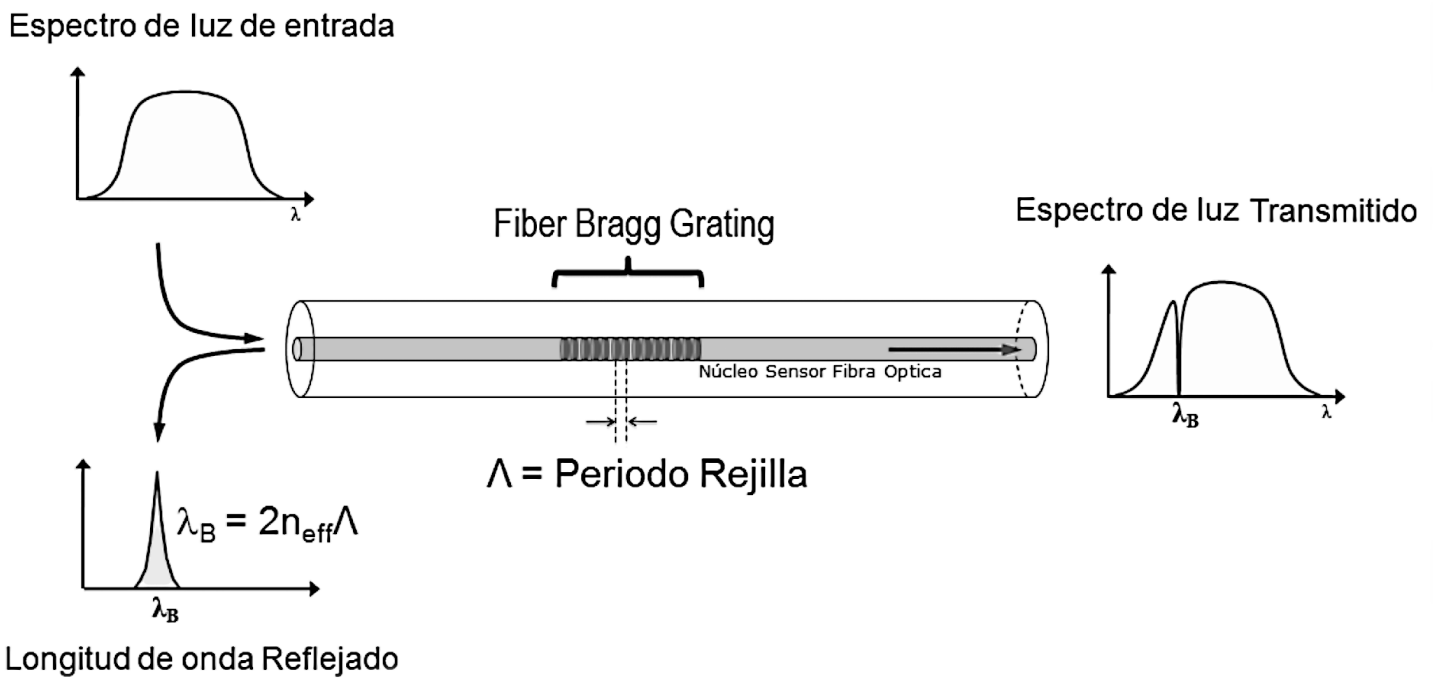

Figura 3. Operación de un sensor de fibra óptica FBG. 
- Laser Barrido de Longitud de onda (WDMSwept Wavelength Laser).

Hoy en día, los más populares interrogadores ópticos WDM son los que utilizan láser de barrido rápido como una fuente de luz en vez de una fuente de amplio espectro. Las ventajas son un mayor alcance (debido a la fuente de energía más alta), una mayor capacidad de sensores (debido a rangos de longitud de onda más amplios de 50 a 100 nm) y la posibilidad de interrogar simultáneamente múltiples fibras, cada una con docenas de sensores.

El número de sensores FBG que puede incorporarse en una sola fibra depende del rango de operación de la longitud de onda de cada sensor y del rango total disponible de longitud de onda del Interrogador óptico. Para asegurar una adecuada operación, se acostumbra dejar un rango aproximado de 4nm para el desplazamiento de la longitud de onda de los sensores de fibra óptica FBG. Los interrogadores típicos proporcionan un rango de medición estándar de $80 \mathrm{~nm}$, por lo que cada serie de sensores FBG puede incorporar hasta 20 sensores por canal del interrogador, siempre y cuando las longitudes de onda reflejadas no se traslapen en el espectro óptico. Debe tenerse el cuidado de seleccionar las longitudes de onda nominales y los rangos para los sensores FBG de forma tal que se garantice que cada sensor opera en un rango espectral único.

La figura 4 muestra un diagrama genérico de la arquitectura interna de un interrogador óptico de Láser de barrido rápido (swept wavelength laser) que usa el método de interrogación WDM para un sistema de sensores de fibra óptica FBG.

\section{Carácterísticas de los Sensores de Fibra Óptica FBG para SHM}

Los sistemas ópticos con sensores de fibra FBG han demostrado ser efectivos en el Monitoreo de la Salud Estructural (SMH, por sus siglas en inglés) de obras civiles en diferentes áreas de aplicación, tales como las industrias de petróleo y gas, generación eléctrica, transporte, aeroespacial e infraestructura civil (Zheng, Naik, Chen, Zhu y Krishnaswamy, 20 I3).

Con respecto a la infraestructura civil, de la cual pueden mencionarse varios tipos, tales como edificios, carreteras, túneles y particularmente puentes, caso en el que se enfoca el presente trabajo, el SMH basado en una plataforma de sensores FBG ha permitido implementar estrategias para la identificación de daños en varios niveles (detección, localización, tipificación, severidad), alertando por anticipado sobre posibles condiciones de riesgo de un puente. Además, si se cuenta con una base de datos y un sistema de monitoreo a largo plazo, es factible evaluar la magnitud de los daños y estimar la vida útil restante de una estructura (Wenzel, 2009).

Entre las principales mediciones que es necesario realizar en Pruebas No Destructivas (NDT, por sus siglas en inglés) para valorar el estado o desempeño de un puente se encuentran deformaciones, desplazamiento, deflexión y vibraciones. Estas mediciones,

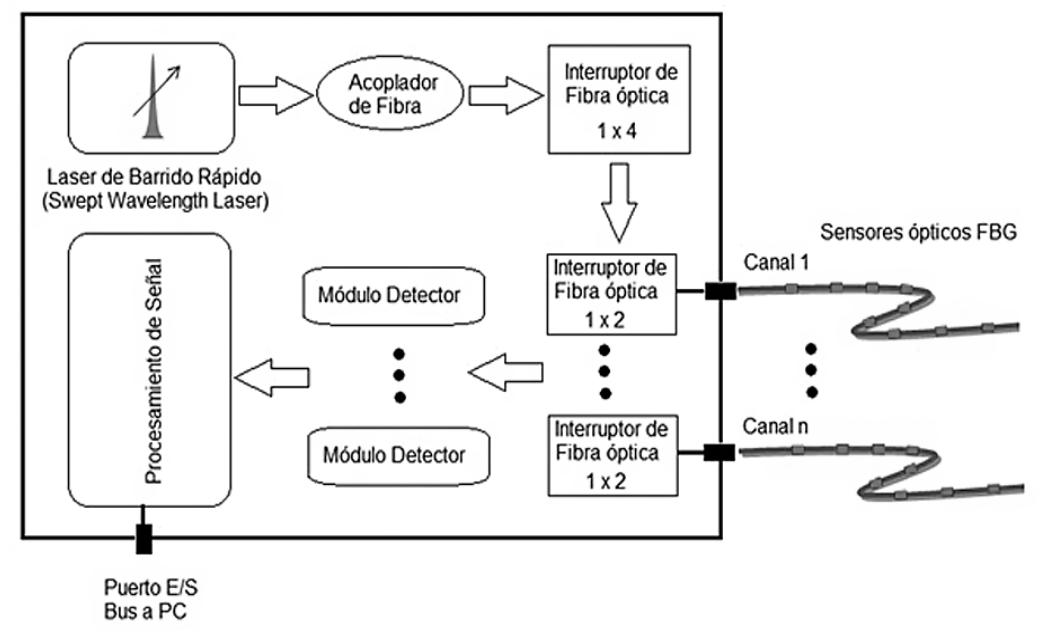

Figura 4. Diagrama Interrogador Laser de barrido rápido para sensores FBG. 
estáticas o dinámicas, pueden relacionarse directamente con los siguientes tipos de sensores de fibra óptica FBG:

- Esfuerzo (strain)

- Temperatura

- Desplazamiento

- Acelerómetros.

A continuación, se expone cada uno de los tipos de sensores FBG mencionados, tomando como referencia el catálogo de productos de la empresa Micron Optics Inc. (20/3).

\section{Medidor de esfuerzo (strain) FBG}

La figura 5 muestra un sensor de esfuerzo (strain) de fibra óptica FBG con compensación de temperatura para medir deformación. Su tamaño aproximado es de $14 \mathrm{~cm}$ de largo por $2,5 \mathrm{~cm}$ de ancho.
El límite de medición es de $\pm 2500 \mu \varepsilon$, con una sensibilidad $\approx 1.2 \mathrm{pm} / \mu \varepsilon$; la vida útil por fatiga de $100 \times 10^{6}$ ciclos a $\pm 2000 \mu \varepsilon$; el rango operativo de temperatura de -40 a $80^{\circ} \mathrm{C}$. En cuanto a las especificaciones ópticas, el rango estándar de longitud de onda se extiende de 1512 a $1586 \mathrm{~nm}$, para un rango operativo de $74 \mathrm{~nm}$, dejando un distanciamiento recomendado de $4 \mathrm{~nm}$ entre la medición de esfuerzo $(\mu \varepsilon)$ y temperatura, para el desplazamiento de las longitudes de onda sin interferencia.

La figura 6 muestra en forma de bosquejo un gráfico sin escala con el espectro de las longitudes de onda de un sensor FBG de esfuerzo, compensado en temperatura, y ejemplifica la correspondiente medición de $\mu \boldsymbol{\varepsilon}$.

\section{Medidor de temperatura FBG}

La figura 7 muestra varios sensores de temperatura de fibra óptica FBG. El os4I00 es un medidor

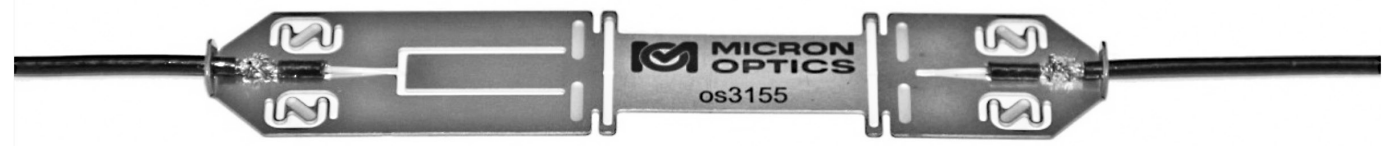

Figura 5. Sensor óptico FBG de esfuerzo: Os3155
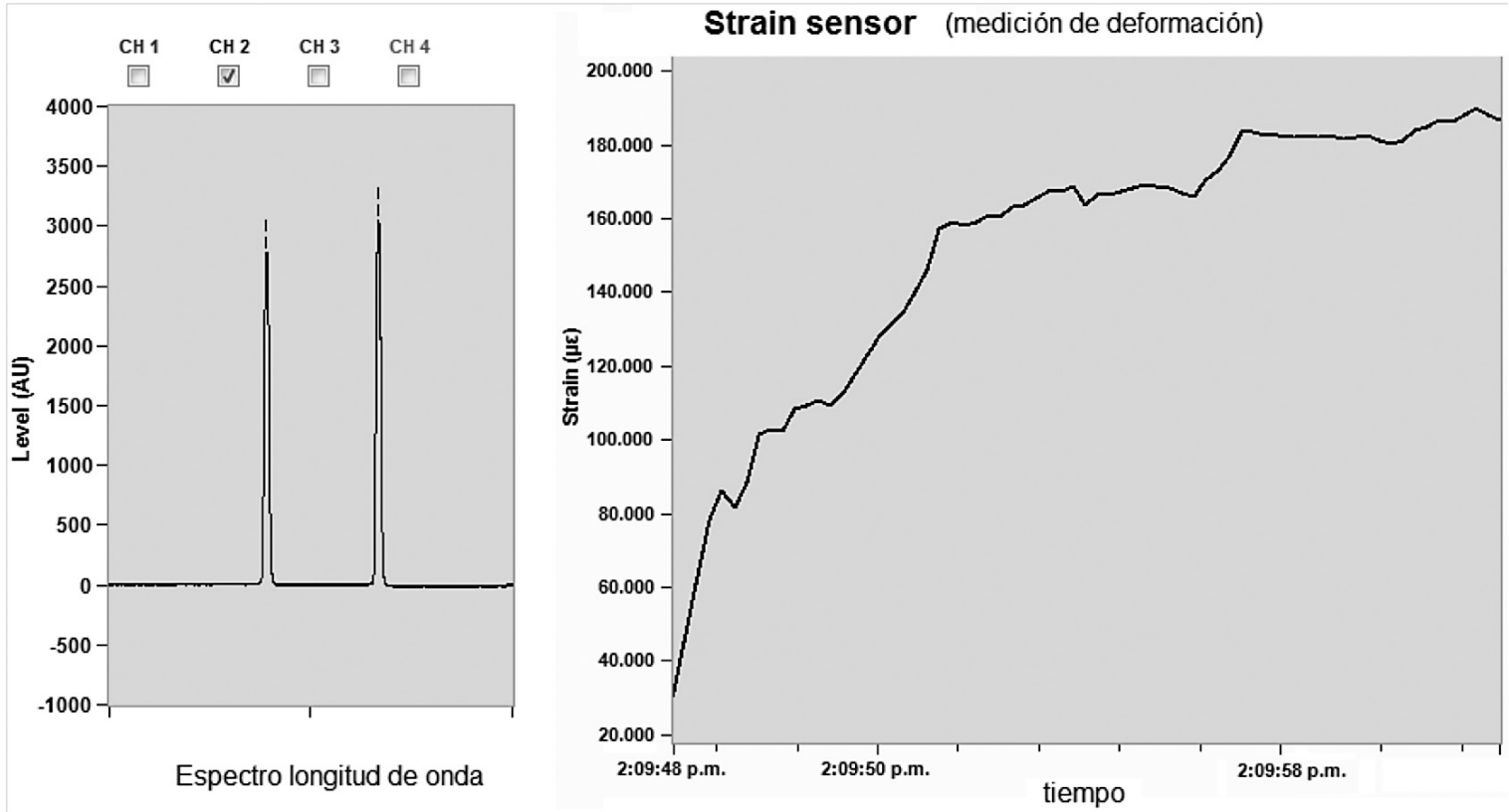

Figura 6. Ejemplo de medición de un sensor óptico FBG de esfuerzo con compensación de temperatura. 

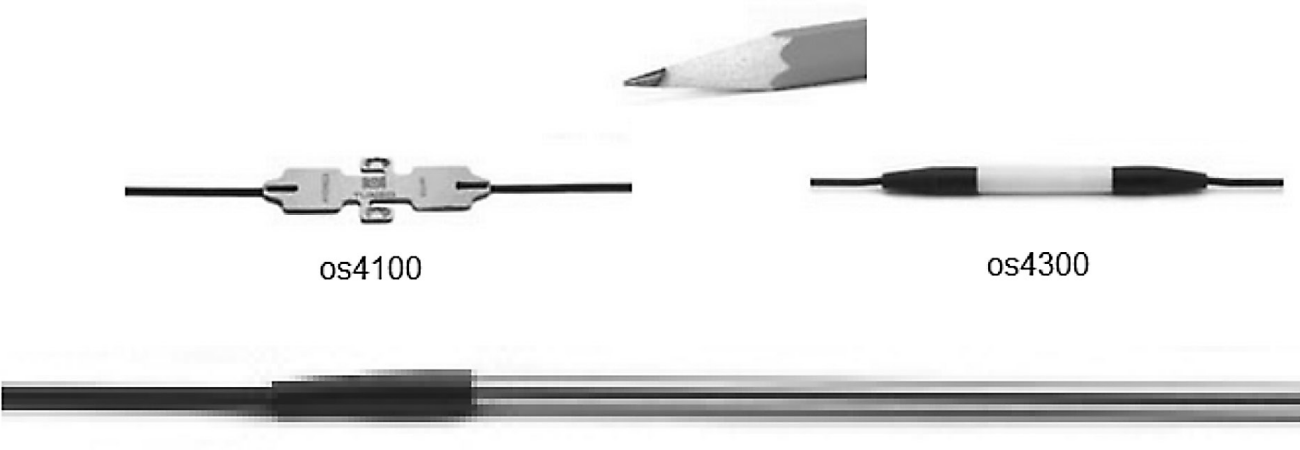

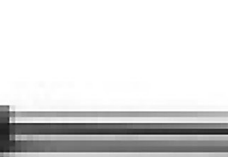
.

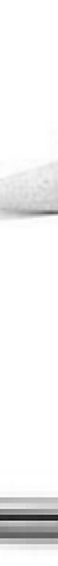

\section{os4230}

Figura 7. Sensores ópticos FBG de temperatura.

diseñado específicamente para proporcionar compensación de temperatura en mediciones de deformación. El os4230 es una sonda de temperatura robusta, cuyo cuerpo es un tubo de acero inoxidable sellado que protege la FBG. El os4300 es un sensor de temperatura no metálico.

Si se toma como referencia la sonda de temperatura para describir las características del sensor, el rango operativo de medición es de - 40 a 120 ${ }^{\circ} \mathrm{C}$ o de -200 a $275{ }^{\circ} \mathrm{C}$, con una sensibilidad $\approx 10 \mathrm{pm} /{ }^{\circ} \mathrm{C}\left( \pm 1,7^{\circ} \mathrm{pm} /{ }^{\circ} \mathrm{C}\right)$. El tiempo de respuesta es de I,5 segundos, con una exactitud a largo plazo de $1{ }^{\circ} \mathrm{C}$ para la calibración estándar. En cuanto a las especificaciones ópticas, el rango estándar de longitud de onda se extiende de 1512 a $1588 \mathrm{~nm}$, para un rango operativo de $76 \mathrm{~nm}$, en intervalos de $4 \mathrm{~nm}$ para el desplazamiento de las longitudes de onda sin interferencia.

La figura 8 muestra, en forma de bosquejo, un gráfico sin escala del espectro de la longitud de onda de un sensor FBG de temperatura y la medición de temperatura correspondiente.

\section{Medidor de desplazamiento FBG}

La figura 9 muestra un sensor de fibra óptica FBG para medir desplazamiento entre dos puntos de medición sobre una superficie de prueba. Su tamaño aproximado es de $15 \mathrm{~cm}$ de largo por $10 \mathrm{~cm}$ de ancho y $5 \mathrm{~cm}$ de alto.

El rango de medición de desplazamiento es de 0 a $50 \mathrm{~mm}$, con una exactitud de $0.03 \mathrm{~mm}$. La velocidad máxima de operación es de $1050 \mathrm{~mm} / \mathrm{seg}$. La vida útil por fatiga es de $10 \times 10^{6}$ ciclos con $40 \mathrm{~mm}$ de carrera. El rango operativo de temperatura es de -40 a $80^{\circ} \mathrm{C}$. En cuanto a las especificaciones ópticas, requiere operar en pares de longitud de onda, fijando un total de $15 \mathrm{~nm}$ por medidor de desplazamiento.

\section{Acelerómetros FBG}

La figura 10 muestra acelerómetros de fibra óptica FBG para la medición de vibraciones. Los acelerómetros FBG se pueden unir a un bloque de montaje para formar configuraciones de uno, dos o tres ejes, como se muestra en la figura.

El propósito de este tipo de sensor es realizar mediciones de la aceleración en grandes estructuras y en un rango de frecuencias bajas, desde 0 a 300 Hertz. Soporta un pico de impacto máximo de $100 \mathrm{~g}$. El rango operativo de temperatura es de -40 a $80^{\circ} \mathrm{C}$. Para el propósito de análisis dinámico, presenta una frecuencia de resonancia de aproximadamente 700 Hertz, con un peso de 28 gramos. Note que la frecuencia de resonancia del dispositivo es superior al rango de medición establecido y por su peso puede considerarse despreciable su contribución en los resultados de un análisis dinámico, aún en componentes de frecuencia de orden superior. En cuanto a las especificaciones ópticas, el rango estándar de longitud de onda se extiende de 1516 a 1588nm, para un rango operativo de $72 \mathrm{~nm}$, con intervalos de al menos $4 \mathrm{~nm}$ entre cada eje.

La figura I I muestra, en forma de bosquejo, un gráfico sin escala del espectro de la longitud de onda de un acelerómetro óptico FBG y la medición de vibraciones correspondiente (Ig equivale $\approx 9.8 \mathrm{~m} / \mathrm{s}^{2}$ ). 


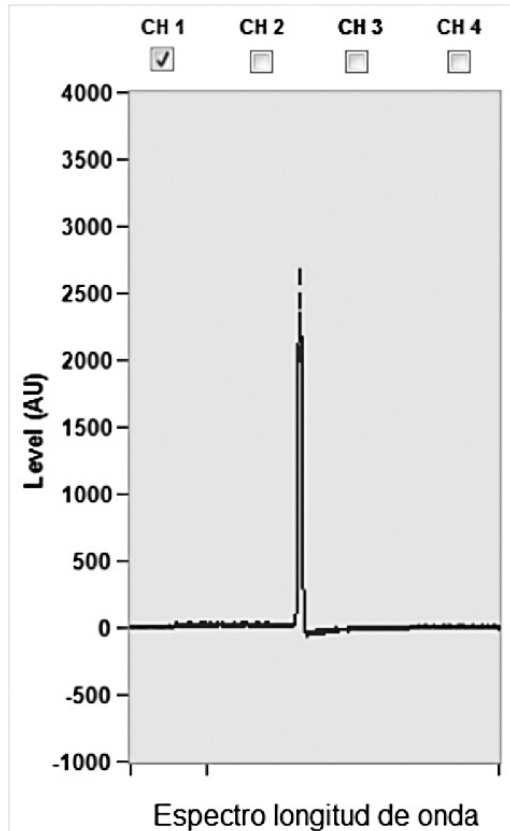

Sensor de Temperatura $\left({ }^{\circ} \mathrm{C}\right)$

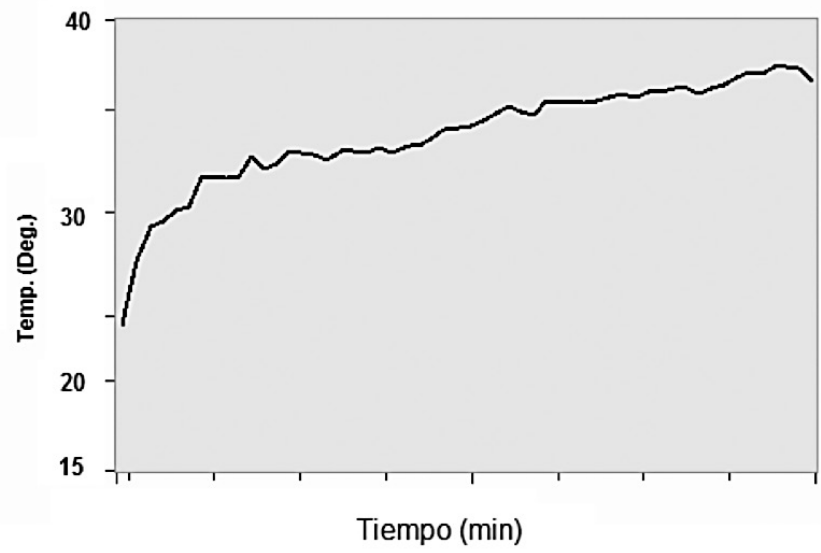

Figura 8. Ejemplo de medición de temperatura de un sensor óptico FBG.

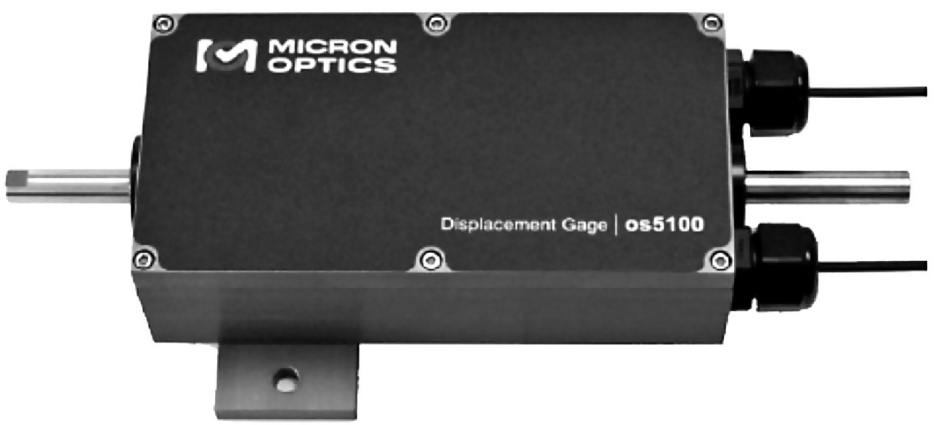

Figura 9. Medidor óptico FBG de desplazamiento: Os5 100.
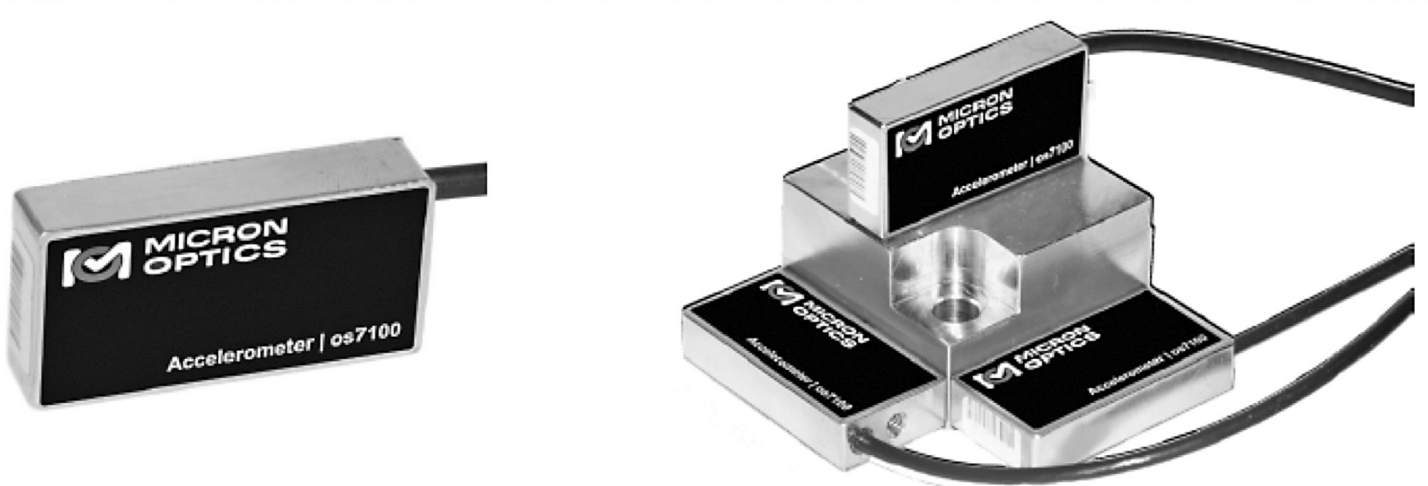

Un solo eje

Montaje Tres ejes

Figura 10. Acelerómetro FBG para medición de vibraciones: Os 7100. 

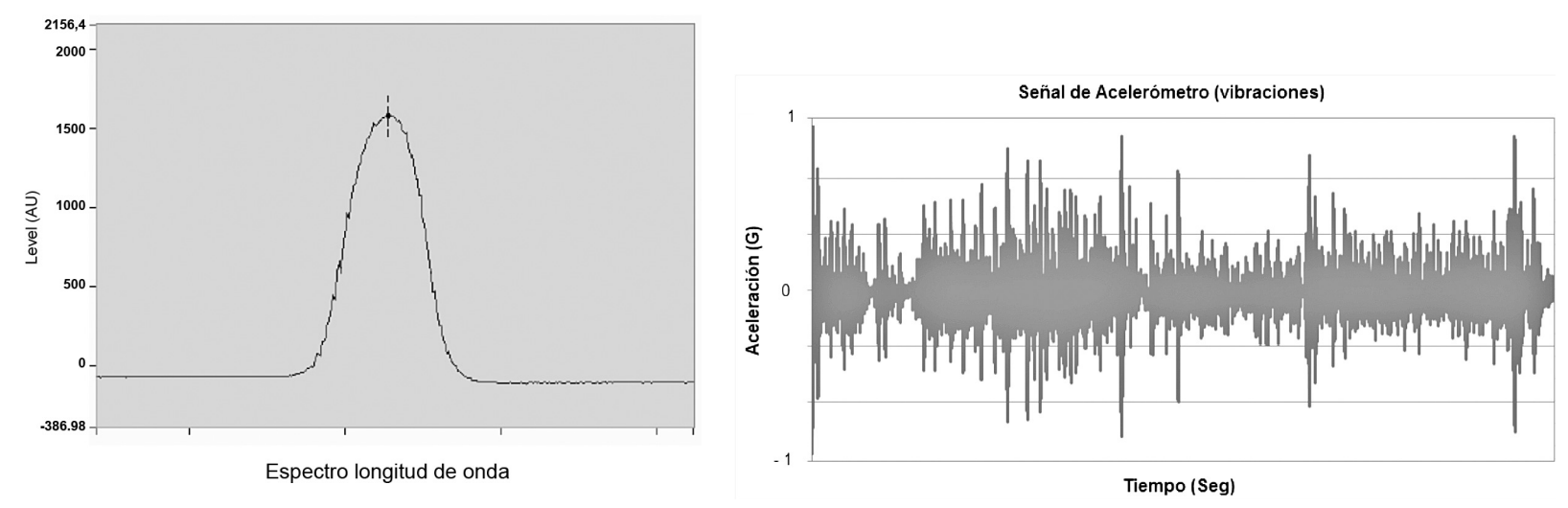

Figura I I. Ejemplo de un acelerómetro FBG para la medición de vibraciones.

\section{Conclusiones}

Se pueden mencionar las siguientes consideraciones de importancia para una adecuada implementación en campo de un sistema de sensores de fibra óptica FBG en aplicaciones reales:

Las mediciones principales con sensores de fibra óptica FBG son deformación (strain) y temperatura.

Un aspecto clave para una exitosa medición con sensores FBG consiste en una adecuada compensación de temperatura.

Existen beneficios inherentes al uso de la tecnología de sensores de fibra óptica FBG, tales como: utiliza un medio no conductivo, eléctricamente pasivo e inmune a la EMl, es posible hacer mediciones a través de distancias largas sin pérdida de precisión de la señal, permite la conexión en serie de múltiples sensores de diferentes tipos en una sola fibra óptica y presenta una vida útil mayor respecto a su contraparte eléctrica.

Los sistemas con sensores ópticos FBG resultan muy útiles en aplicaciones que no pueden ser fácilmente implementadas o no resulta práctico realizarlas con sensores eléctricos convencionales, especialmente cuando se requiere una gran cantidad de sensores y la complejidad de la ingeniería necesaria para la solución aumenta con el número de sensores.

Un sistema basado en sensores de fibra óptica FBG para el monitoreo de la salud estructural de los puentes, y en general de obra civil, proporciona información suficiente para valorar el comportamiento estructural, identificar condiciones de riesgo y estimar la vida útil restante de una estructura.

\section{Bibliografía}

Davis, M. A., Bellemore, D. G., Berkoff,T. A., Kersey, A. D., Putnam, M. A., Idriss, R. L. y Kodinduma, M. (1996). Fiber optic sensor system for bridge monitoring with both static load and dynamic modal sensing capabilities. doi: | 0.1 | | /7/ | 2.259 | 40

Hill, K. O., Fujii, Y., Johnson, D. C. y Kawasaki, B. S. (1978). Photosensitivity in Optical Fiber Waveguides: Application to Reflective Filter Waveguide. Appl. Phys. Lett., 32, 647-649.

Kumar, K. y Madhav, V. (20I0). Review on developments in fiber optical sensors and applications. Obtenido de http://spiedigitallibrary.org

Micron Optics, Inc. (2012). Optical fiber sensor guide: fundamentals and applications. Obtenido de http://www.micronoptics. com/technology/document_library/index.php

Micron Optics, Inc. (2013). Optical fiber sensor datasheet. Obtenido de http://www.micronoptics.com/products/sensing_solutions/sensors/

National Instruments, Inc. ( 15 de noviembre, 20 I0). Fundamentos de la Detección Óptica FBG. Obtenido de http://www. ni.com/white-paper/ / | 82 |/es/

National Instruments, Inc. ( 19 de abril, 20 I I ). FBG Optical Sensing: A New Alternative for Challenging Strain Measurements. Obtenido de http://www.ni.com/white-paper/ | 2338/en/

National Instruments, Inc. (30 de junio, 201 I). Overview of Fiber Optic Sensing Technologies. Obtenido de http://www.ni.com/ white-paper/ / 2953/en/

Tomasi, W. (2003). Comunicaciones con fibra óptica. En Pearson Educación de México (Ed.), Sistemas de comunicaciones eléctricas (pp. 422-465). México: Pearson Educación Inc.

Wenzel, H. (2009). Health Monitoring of Bridges. Viena: John Wiley \& Sons.

Zheng, S., Naik, G., Chen, Z., Zhu, Y. y Krishnaswamy, S. (20I3). Sensing platforms for structural health monitoring. Disponible en http://spiedigitallibrary.org 